\title{
New Galactic Candidate Luminous Blue Variables and Wolf-Rayet Stars
}

\author{
Guy S. Stringfellow ${ }^{1}$, Vasilii V. Gvaramadze ${ }^{2}$, Yuri Beletsky ${ }^{3}$, \\ and Alexei Y. Kniazev ${ }^{4}$ \\ ${ }^{1}$ Center for Astrophysics and Space Astronomy, \\ University of Colorado, 389 UCB, Boulder, CO 80309-0389, USA \\ email: Guy.Stringfellow@colorado.edu \\ ${ }^{2}$ Sternberg Astronomical Institute, Moscow State University, Universitetskij Pr. 13, Moscow \\ 119992, Russia \\ email: vgvaram@iki.rssi.ru \\ ${ }^{3}$ European Southern Observatory, Alonso de Cordova 3107, Santiago, Chile \\ email: ybialets@eso.org \\ ${ }^{4}$ South African Astronomical Observatory, PO Box 9, 7935 Observatory, Cape Town, South \\ Africa \\ email: akniazev@saao.ac.za
}

\begin{abstract}
We have undertaken a near-infrared spectral survey of stars associated with compact mid-IR shells recently revealed by the MIPSGAL $(24 \mu \mathrm{m})$ and GLIMPSE $(8 \mu \mathrm{m})$ Spitzer surveys, whose morphologies are typical of circumstellar shells produced by massive evolved stars. Through spectral similarity with known Luminous Blue Variable (LBV) and Wolf-Rayet (WR) stars, a large population of candidate LBVs (cLBVs) and a smaller number of new WR stars are being discovered. This significantly increases the Galactic cLBV population and confirms that nebulae are inherent to most (if not all) objects of this class.
\end{abstract}

Keywords. stars: emission-line, Be, stars: mass loss, stars: winds, outflows, stars: Wolf-Rayet

\section{Introduction}

Despite intensive search efforts over the last several decades the Galactic Luminous Blue Variable (LBV) population has remained sparse. This paucity is difficult to reconcile with our understanding of stellar evolution of the most massive stars. Until the last year or two there were only 12 confirmed Galactic LBVs known, and 23 candidate-LBVs (Clark et al. 2005). LBVs display rather unique rich infrared emission line spectra, including contributions from H, He, Mg II, Na I, and Fe II. Visual inspection of the Spitzer GLIMPSE (Benjamin et al. 2003) and MIPSGAL (Carey et al. 2009) Galactic plane surveys have produced catalogues of previously unknown $8 \mu \mathrm{m}$ and $24 \mu \mathrm{m}$ nebulae with concentric point sources that can be traced back to 2MASS $K$-band or even optical sources as possible progenitors of the associated nebulae (Gvaramadze et al. 2010a, Wachter et al. 2010), especially with new imaging (Stringfellow et al. 2012). We are conducting a nearIR spectral survey to identify new cLBVs and WRs that produced these shells.

\section{Observations and Results}

We have obtained spectra of $\sim 50$ stars associated with newly discovered mid-IR nebulae using SpeX on the NASA IRTF 3m, Triplespec on APO $3.5 \mathrm{~m}$ and Palomar Hale 5m, and ISAAC on the ESO-VLT. A few of the $K$-band spectra are shown in Figure 1 . The 

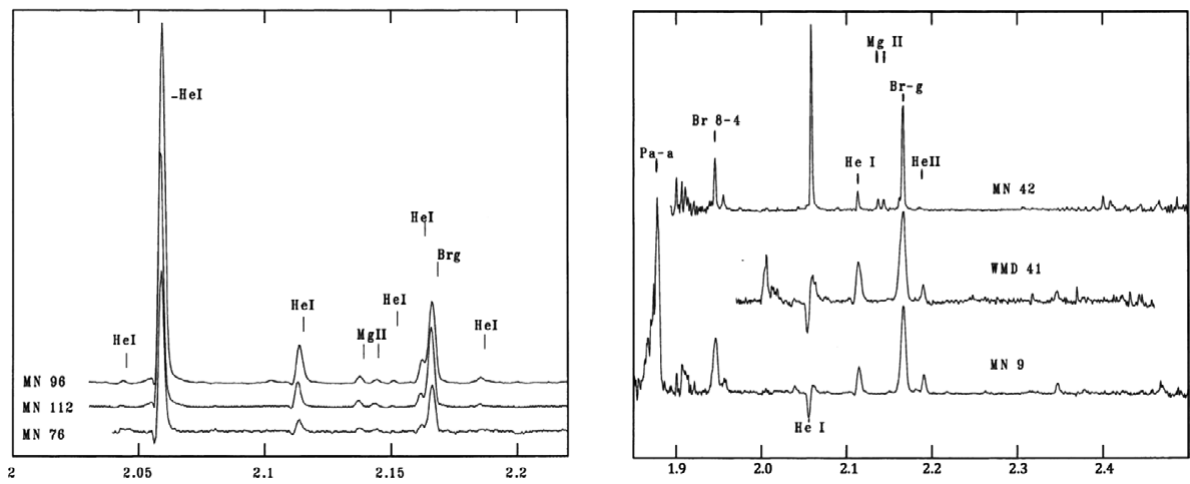

Figure 1. Normalized $K$-band spectra of newly identified cLBVs and WRs. See Figure 1 of Stringfellow et al. 2012 for images identifying MN 96, including optical recovery in the $I$-band.

left panel shows three newly identified cLBVs that have Fe II emission absent in their IR spectra. Prominent line emission arise from $\mathrm{He}$ I, $\mathrm{Br} \gamma$, and $\mathrm{Mg}$ II. A spectrum of MN 96 (WMD 54, Wachter et al. 2010), is discussed in Wachter et al. (2011), who notes the similarity between both LBV and WR late-type WN spectra for this particular star. Our spectrum clearly indicates the absence of any He II $2.189 \mu \mathrm{m}$ line emission. Comparison of an optical spectrum of MN 112 with that of P Cyg rendered classification as a cLBV (Gvaramadze et al. 2010b); both spectra display numerous optical Fe III lines, but no Fe II line emission. The absence of the $2.089 \mu \mathrm{m}$ Fe II line in the MN $112 \mathrm{~K}$-band spectrum is consistent with a higher temperature in this line emitting region. MN 76 (WMD 38) was classified as a Be star (Wachter et al. 2011), though no spectrum was shown. Clearly the $K$-band spectra for these three stars - MN 96, MN 112, and MN 76 - are nearly identical (barring small differences in line widths and strengths), and should render the same IR spectral classification. These stars could be transitional between the LBV and late-WN stars, or have spectral types varying between minimum contraction to maximum expansion, corresponding to hot and cool temperature phases, respectively. VLT spectra for two WRs, WMD 41 (WN8-9h) and MN 9 (WN7-9h), are displayed in the right panel of Figure 1 along with the VLT spectrum for the cLBV MN 42 (WMD 15). The WR spectra lack Mg II emission and display broader $\mathrm{H}$ and He lines than the cLBVs. MN 42 was classified as B[e]/LBV by Wachter et al. (2011) though no spectrum was shown. MN 42 resembles those cLBVs shown in the left panel, lacking Fe II emission, strengthening the case that the FeII-deficient cLBVs may be transitioning to late-WN stars. We designate MN 42, MN 76, MN 96, and MN 112 as currently FeII-deficient cLBVs.

GSS thanks the AAS for receipt of an ITG and SmRG, and the IAU for support.

\section{References}

Benjamin, R. A., et al., 2003, PASP, 115, 953

Carey, S. J., et al., 2009, PASP, 121, 76

Clark, J. S., Larionov, V. M., \& Arkharov, A. 2005, AA, 435, 239

Gvaramadze, V. V., Kniazev, A. Y., \& Fabrika, S. 2010a, MNRAS, 405, 1047

Gvaramadze, V. V., et al., 2010b, MNRAS, 405, 520

Stringfellow, G. S., et al., 2012, in Four Decades of Research on Massive Stars: A Scientific Symposium in Honour of Anthony F.J. Moffat, ASP Vol., eds L. Drissen, N. St-Louis, C. Robert, \& A.F.J. Moffat (in press)

Wachter, S., et al., 2010, AJ, 139, 2330

Wachter, S., et al., 2011, BSRSL, 80, 291 